\title{
O Método De Rayleigh-Ritz aplicado a um Problema de Deflexão de Viga utilizando o Matlab
}

\author{
Yuri Elias Rodrigues* Daniel da Silva Rodrigues** \\ Pontifícia Universidade Católica do Rio Grande do Sul - Faculdade de Matemática \\ 90619-900, Av. Ipiranga, 6681 - Partenon - Porto Alegre, RS \\ E-mail: yuri.rodrigues@acad.pucrs.br \\ Eliete Biasotto Hauser \\ Pontifícia Universidade Católica do Rio Grande do Sul - Faculdade de Matemática \\ 90619-900, Av. Ipiranga, 6681 - Partenon - Porto Alegre, RS \\ E-mail: eliete@pucrs.br
}

\section{RESUMO}

Este trabalho tem como objetivo avaliar os resultados do Método de Rayleigh-Ritz (MRR) aplicado ao seguinte Problema de Valor de Contorno (PVC) que modela a deflexão de uma viga apoiada sobre seus extremos:

$$
\left\{\begin{array}{l}
-\frac{d}{d x}\left(p(x) \frac{d y}{d x}\right)+q(x) y=f(x), \quad 0 \leq x \leq 1 \\
y(0)=y(1)=0 .
\end{array}\right.
$$

A solução exata de (1), $y(x)$, é aproximada por uma combinação linear de funções de base $\phi_{i}$.

$$
\Phi(x)=\sum_{i=1}^{n} c_{i} \phi_{i}(x)
$$

As funções $\phi_{i}$ devem satisfazer as condições de fronteira e serem linearmente independentes. Para atender esta imposição selecionamos como funções de base, as funções lineares por partes expressas por:

$$
\phi_{i}(x)=\left\{\begin{array}{r}
0, \text { se } 0 \leq x \leq x_{i-1} \\
\frac{x-x_{i-1}}{h_{i-1}}, \text { se } x_{i-1}<x \leq x_{i} \\
\frac{x_{i+1}-x}{h_{i}}, \text { se } x_{i}<x \leq x_{i+1} \\
0, \text { se } x_{i+1}<x \leq 1
\end{array}\right.
$$

ilustradas graficamente na figura 1.

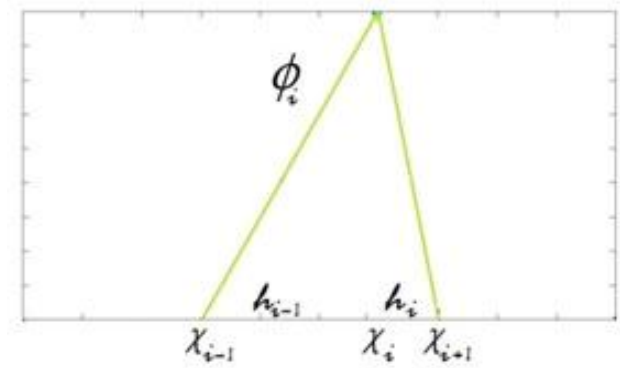

Figura 1. Representação gráfica de $\phi_{i}(x)$.

Em (3) temos que $h_{i}=x_{i+1}-x_{i}$. A escolha deste espaçamento não constante, possibilita que a aproximação seja melhorada utilizando o mesmo número nodos.

* Bolsista de Iniciação Científica PIBIC/CNPq

** Bolsista de Iniciação Científica BPA/PUCRS 
Para determinar os coeficientes $c_{i}$ é necessário, de acordo com [3], minimizar o funcional associado ao PVC (1), portanto, de forma geral temos que:

$$
I(y)=\int_{0}^{1}\left\{p(x)\left[\frac{d y(x)}{d x}\right]^{2}+q(x)[y(x)]^{2}-2 f(x) y(x)\right\} d x .
$$

Para tanto, substituímos (2) na equação (4) obtendo:

$$
I=\int_{0}^{1}\left\{p(x)\left[\sum_{i=1}^{n} \frac{d \phi_{i}(x)}{d x} c_{i}\right]^{2}+q(x)\left[\sum_{i=1}^{n} \phi_{i}(x) c_{i}\right]^{2}-2 f(x) \sum_{i=1}^{n} \phi_{i}(x) c_{i}\right\} d x
$$

O valor mínimo do funcional ocorre quando sua derivada parcial em relação á $c_{i}$ é igual a 0 , ou seja

Então,

$$
\frac{\partial I}{\partial c_{j}}=0
$$

$$
\int_{0}^{1} f(x) \phi_{j}(x) d x=\sum_{i=1}^{n} \int_{0}^{1}\left\{p(x) \frac{d \phi_{i}(x)}{d x} \frac{d \phi_{j}(x)}{d x}+q(x) \phi_{i}(x) \phi_{j}(x)\right\} d x .
$$

A equação (7) pode ser representada matricialmente na forma

$$
\text { A. } C=B \text {, }
$$

onde $A$ é uma matriz tridiagonal de ordem $n \times n$, e seus elementos são expressos por

$$
a_{i j}=\int_{0}^{1}\left\{p(x) \frac{d \phi_{i}(x)}{d x} \frac{d \phi_{j}(x)}{d x}+q(x) \phi_{i}(x) \phi_{j}(x)\right\} d x
$$

Os elementos do vetor $B$ são dados por

$$
b_{i}=\int_{0}^{1} f(x) \phi_{i}(x) d x .
$$

Para validar a solução aproximada de (1) consideremos o PVC que tem solução conhecida:

$$
\left\{\begin{array}{l}
-\frac{d}{d x}\left(e^{x} \frac{d y}{d x}\right)+e^{x} y=x+(2-x) e^{x}, \quad 0 \leq x \leq 1 \\
y(0)=y(1)=0 .
\end{array}\right.
$$

O qual tem solução exata

$$
y(x)=(x-1)\left(e^{-x}-1\right) .
$$

O algoritmo MRR foi implementado em linguagem de programação do software Matlab. Com $n=2, n=5$ e $n=10$, e $h$ assumindo respectivamente os valores $1 / 3,1 / 6$ e $1 / 11$, ilustramos graficamente os resultados na figura 3, em que: a aproximação obtida pelo MRR, $\Phi(x)$, esta em azul; a solução exata, $y(x)$, esta em vermelho; o erro absoluto, $E(x)=|y(x)-\Phi(x)|$, esta em verde.
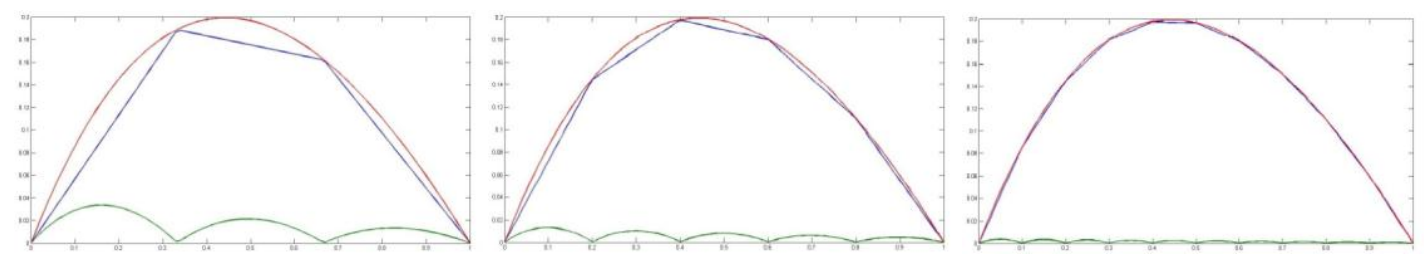

Figura 3. $y(x), \Phi(x)$ e $E(x), \operatorname{com} n=2, n=5$ e $n=10$. 
Na tabela 1, são apresentados os valores dos erros absolutos para as simulações ilustradas na figura 3, e seu padrão sugere a convergência de $\Phi(x)$ para $y(x)$, à medida que aumentarmos a quantidade de nodos.

\begin{tabular}{|c|c|c|c|}
\hline$x_{i}$ & $n_{1}=2$ & $n_{2}=5$ & $n_{3}=10$ \\
\hline 0 & 0 & 0 & 0 \\
\hline 0.1 & 0.029025685184959 & 0.013232147308362 & 0.000078048779597 \\
\hline 0.2 & 0.031774120372259 & 0.000187044619065 & 0.000148785680326 \\
\hline 0.3 & 0.011565329774764 & 0.010275050194529 & 0.000201733484602 \\
\hline 0.4 & 0.014444794880438 & 0.000331934640629 & 0.000231318797700 \\
\hline 0.5 & 0.021429919308394 & 0.007923088068827 & 0.000235833486994 \\
\hline 0.6 & 0.013229021389989 & 0.000328219150663 & 0.000216594059407 \\
\hline 0.7 & 0.032409319228047 & 0.005978229257140 & 0.000177264949847 \\
\hline 0.8 & 0.024737309003027 & 0.000188974377407 & 0.000123317302782 \\
\hline 0.9 & 0.026966270242601 & 0.004370417626366 & 0.000615995439889 \\
\hline 1 & 0 & 0 & 0 \\
\hline
\end{tabular}

Tabela 1. Erros absolutos, $E(x)$.

Adicionalmente podemos verificar nas simulações que o erro é maior no centro do intervalo, portanto a liberdade de escolha de cada $h_{i}$ pode ser usada para limitar o erro absoluto máximo.

Por exemplo, para $n=2$ com $h_{0}=0.26222, h_{1}=0.32799$ e $h_{2}=0.58999$, o erro absoluto máximo é aproximadamente 0.0215 .

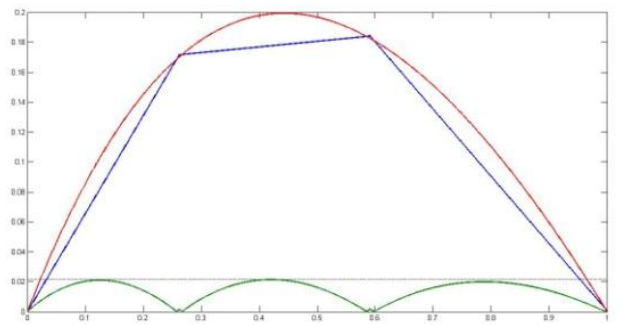

Figura 4. $y(x), \Phi(x)$ e $E(x)$ para $n=2$ e $h_{0}, h_{1}$ e $h_{2}$.

Palavras-chave: Método de Rayleigh-Ritz, Equações Diferenciais Ordinárias, Problemas de Valor de Contorno

\section{Referências}

[1] A.W. Leissa, "The historical bases of the Rayleigh and Ritz methods", Journal of Sound and Vibration 287.pp. $961-978,2005$.

[2] A. E. Assan, “Análise Numérica”, São Paulo, SP. Ed. Thomson. 2003.

[3] L. Burden, e D. Faires, "Numerical analysis", $9^{\circ}$ edition. Cengage Learning, pp. 673-679. 2011.

[4] J. Júdice, "Sistemas de Equações Lineares", Coimbra, Portugal, Departamento de Matemática da Universidade de Coimbra, Capítulo 5, 1996

[5] M. Schultz, "Spline Analyses”, Prentice Hall, pp. 88-89, 1973.

[6] MATHWORKS, "User's Guide - The Student Edition of Matlab, The Ultimate Computing Environment for Technical Education”, Prentice Hall, 1995. 